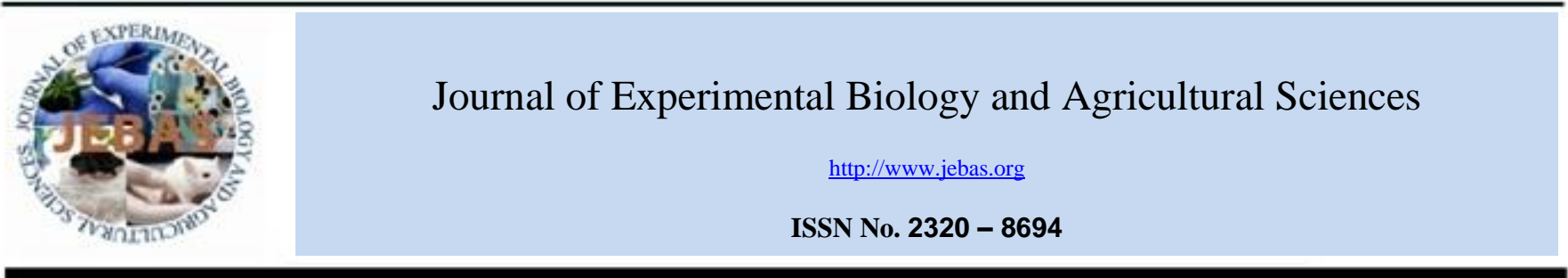

\title{
PERFORMANCE OF RICE GENOTYPES UNDER DIFFERENT SEED RATES AND MICRONUTRIENTS IN DIRECT SEEDED CONDITION UNDER TUNGABHADRA IRRIGATION COMMAND
}

\author{
University of Agriculture Sciences, Raichur, Karnataka (India) \\ Received - May 11, 2018; Revision - June 17, 2018; Accepted - July 21, 2018 \\ Available Online - August 10, 2018 \\ DOI: http://dx.doi.org/10.18006/2018.6(4).701.706
}

Shubha GV*, BM Chittapur, A.S. Halepyati, Veeresh H

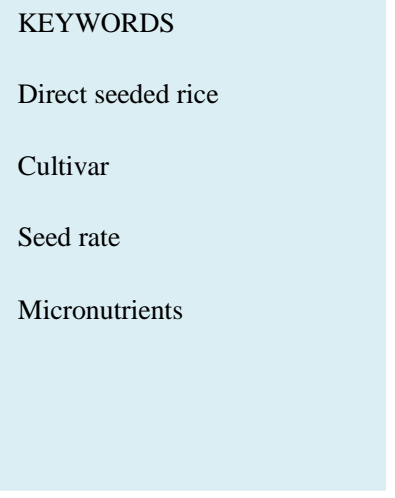

\begin{abstract}
The present investigation was conducted during kharif season of 2015 and 2016 at agriculture research station, Dhadesugur, University of agricultural sciences, Raichur to optimizing the performance of genotypes to different seed rates and micronutrients in direct seeded condition under Tungabhadra irrigation command. Treatments were tested using split-split plot design with three replications keeping varieties in main-plots, seed rates in sub-plots and micronutrient levels in sub-sub plot. Among various tested combination, higher grain yield was reported in cv. MTU 1010 (5917 kg ha ${ }^{-1}$ ) and this was followed by cvs. GGV 0501 (5115 kg ha $\left.{ }^{-1}\right)$ and BPT 5204 (4663 kg ha ${ }^{-1}$ ). Net returns of Rs. 78,084 was higher with cv. MTU 1010 sown using $30 \mathrm{~kg} \mathrm{ha}^{-1}$ and applied with both $\mathrm{FeSO}_{4}$ and $\mathrm{ZnSO}_{4}$ this was followed by cv. BPT 5204 (Rs.76,533 ha ${ }^{-1}$ ) at $30 \mathrm{~kg} \mathrm{ha}^{-1}$ and applied with both $\mathrm{FeSO}_{4}$ and $\mathrm{ZnSO}_{4}$.Similar trend was obtained in Benefit cost ratio of 2.47 and 2.43.
\end{abstract}

* Corresponding author

E-mail: agshubha@gmail.com (Shubha GV)

Peer review under responsibility of Journal of Experimental Biology and Agricultural Sciences.

Production and Hosting by Horizon Publisher India [HPI] (http://www.horizonpublisherindia.in/).

All rights reserved.
All the article published by Journal of Experimental Biology and Agricultural Sciences is licensed under a Creative Commons Attribution-NonCommercial 4.0 International License Based on a work at www.jebas.org.

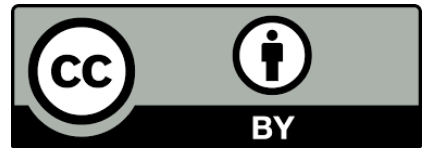




\section{Introduction}

Rice is one of the most important staple food crops, in Asia, Africa and Latin America, millions of people living in the tropics and subtropics depend on it. In these areas, population growth rate is rapid and will likely to remain high at least for the next decade (FAO, 2017). In the world, rice is grown on $158.0 \mathrm{~m} \mathrm{ha}^{-1}$ with a production of $746.9 \mathrm{mt}$ and productivity of $3889 \mathrm{~kg} \mathrm{ha}^{-1}$ (FAO, 2014). In India, rice continues to hold the key to sustain food production by contributing $20-25 \%$ and assures food security for more than half of the total population (FAO, 2017). To meet the challenge of producing more rice from suitable lands, it is necessary to use rice varieties with higher yield potential and greater yield stability (Khush, 2004). More than two billion people are getting 60-70 per cent of their energy requirement from rice and its derived products in Asia (Rekha et al., 2015). Rice crop is grown by many ways and these are depending upon availability of resources. Due to resource constraints, especially water and laborers, direct seeding under dry condition is now emerging new trend in rice cultivation. Direct-seeding of rice has the potential to provide several benefits to farmers and the environment over conventional practices of puddling and transplanting. Direct seeding (both wet and dry) avoids nursery raising, seedling uprooting, puddling and transplanting, and thus reduces the labor requirement. Therefore, developing new cultivars and their testing for adoptability and yield would be a continuous job. To realize the maximum possible benefits of rice crop and to obtain higher yield, it is essential to adopt recommended package of agronomic practices for successful cultivation of rice. Among the various cultural practices selection of variety, optimum seed rate and application of micronutrients are most important for yield maximization. The present investigation entitled Performance of genotypes to different seed rates and micronutrients in direct seeded condition under Tungabhadra irrigation command.

\section{Material and Methods}

Present experiment was conducted during the kharif season of 2015 and 2016 at agriculture research station, Dhadesugur, University of agricultural sciences, Raichur, India. Geographically it is situated on the latitude of $15^{\circ} 6^{\prime}$ North, longitude of $76^{\circ} 8$ ' East and at an elevation of 358 meters above mean sea level. Soil of experimental field was medium black and clay loam in texture. The soil was neutral in reaction (pH 7.20 and 7.04) and relatively high in soluble salts (1.13 and $\left.1.08 \mathrm{dS} \mathrm{m}^{-1}\right)$. The soil was high in organic carbon $(0.86$ and 0.84$)$, medium in available nitrogen $(286.0$ and $280.0 \mathrm{~kg}$ $\left.\mathrm{ha}^{-1}\right)$ and available $\mathrm{P}_{2} \mathrm{O}_{5}\left(22.2\right.$ and $\left.27.2 \mathrm{~kg} \mathrm{ha}^{-1}\right)$, while it was high in $\mathrm{K}_{2} \mathrm{O}$ (427.0 and $438.5 \mathrm{~kg} \mathrm{ha}^{-1}$ ). The DTPA extractable micronutrient content revealed deficient zinc $(0.63$ and $0.61 \mathrm{mg} \mathrm{kg}^{-1}$ ) and iron (4.41 and $4.40 \mathrm{mg} \mathrm{kg}^{-1}$ ) during kharif 2015 and kharif 2016 respectively (Table1). The experiment was laid out in split split plot design keeping cultivars in main plot treatments $\mathrm{V}_{1^{-}}$Gangavathi sona (GGV 0501 ), $\mathrm{V}_{2^{-}}$MTU 1010 and $\mathrm{V}_{3}$ - BPT -5204 , seed rates in sub plot treatments: $\mathrm{S}_{1-}$ $25 \mathrm{~kg} \mathrm{ha}^{-1}, \mathrm{~S}_{2^{-}} 30 \mathrm{~kg} \mathrm{ha}^{-1}$ and $\mathrm{S}_{3^{-}} 35 \mathrm{~kg} \mathrm{ha}^{-1}$, and in sub-sub plot treatments: $\mathrm{M}_{1-}$ Control (no micronutrients), $\mathrm{M}_{2-}-\mathrm{FeSO}_{4}$ (Soil application@25 kg ha ${ }^{-1}+$ foliar application @ 0.5\% twice at 15 and 30 DAS), $\mathrm{M}_{3}-\mathrm{ZnSO}_{4}$ (Soil application @ 25 $\mathrm{kg} \mathrm{ha}^{-1}+$ foliar application @ $0.5 \%$ twice at 15 and 30 DAS) and

Table 1 Physico-chemical properties of the study area soil

\begin{tabular}{|clcccl|}
\hline S. N. & \multicolumn{1}{c}{ Parameters } & 2015 & 2016 & Ratings & \multicolumn{1}{l|}{ Methodology } \\
\hline 1 & $\mathrm{pH}_{(1: 2.5)}$ & 7.20 & 7.04 & Neutral & $\mathrm{pH}$ meter (Piper, 1966) \\
\hline 2 & $\mathrm{EC}_{(1: 2.5)}\left(\mathrm{dSm}^{-1}\right)$ & 1.13 & 1.08 & High & Conductivity bridge (Jackson, 1967) \\
\hline 3 & Organic carbon (\%) & 0.86 & 0.84 & High & Wet oxidation method (Jackson, 1967) \\
\hline 4 & Available $\mathrm{N}_{\left(\mathrm{kg} \mathrm{ha}^{-1}\right)}$ & 286 & 280 & Medium & Alkaline permanganate method (Subbaiah \& Asija, 1956) \\
\hline 5 & Available $\mathrm{P}_{2} \mathrm{O}_{5}\left(\mathrm{~kg} \mathrm{ha}^{-1}\right)$ & 22.2 & 27.2 & Medium & Olsen's method (Jackson, 1967) \\
\hline 6 & Available $\mathrm{K}_{2}{\mathrm{O}\left(\mathrm{kgha}^{-1}\right)}^{2}$ & 427 & 438.5 & High & Flame photometry method (Jackson, 1967) \\
\hline 9 & DTPA Fe $\left(\mathrm{mg} \mathrm{kg}^{-1}\right)$ & 4.41 & 4.40 & Deficient & DTPA extractable micronutrients method (Lindsay \& Norvell, 1978) \\
\hline 10 & DTPA Zn $\left(\mathrm{mg} \mathrm{kg}^{-1}\right)$ & 0.63 & 0.61 & Deficient & DTPA extractable micronutrients method (Lindsay \& Norvell, 1978) \\
\hline
\end{tabular}


Table 2 Effect of genotypes, seed rates and micronutrients grain yield $\left(\mathrm{kg} \mathrm{ha}^{-1}\right)$ under direct seeded condition during rainy season

\begin{tabular}{|c|c|c|c|c|c|c|c|c|c|c|c|c|c|}
\hline \multirow{2}{*}{\multicolumn{2}{|c|}{$\mathrm{V} \times \mathrm{S} \times \mathrm{M}$}} & \multicolumn{4}{|c|}{ 2014-15 } & \multicolumn{4}{|c|}{ 2015-16 } & \multicolumn{4}{|c|}{ Pooled } \\
\hline & & $\mathrm{V}_{1}$ & $\mathrm{~V}_{2}$ & $\mathrm{~V}_{3}$ & S x M & $\mathrm{V}_{1}$ & $\mathrm{~V}_{2}$ & $\mathrm{~V}_{3}$ & $S \times M$ & $\mathrm{~V}_{1}$ & $\mathrm{~V}_{2}$ & $\mathrm{~V}_{3}$ & S x M \\
\hline \multirow{4}{*}{$\mathbf{S}_{1}$} & $M_{1}$ & $5014^{\mathrm{k}-\mathrm{s}}$ & $5664^{\mathrm{d}-\mathrm{n}}$ & $4305^{r-t}$ & $4994^{e-g}$ & $4016^{\mathrm{i}-\mathrm{n}}$ & $4734^{\mathrm{e}-1}$ & $3567^{1-n}$ & $4106^{\mathrm{f}}$ & $4515^{\mathrm{m}-\mathrm{p}}$ & $5199^{\mathrm{f}-\mathrm{m}}$ & $3136^{\mathrm{q}}$ & $4283^{f}$ \\
\hline & $\mathbf{M}_{2}$ & $5349^{g-p}$ & $5762^{\mathrm{c}-1}$ & $4603^{\mathrm{p}-\mathrm{t}}$ & $5238^{d-f}$ & $4083^{\mathrm{h}-\mathrm{n}}$ & $5049^{c-j}$ & $3854^{j-n}$ & $4329^{\mathrm{ef}}$ & $4716^{\mathrm{i}-\mathrm{o}}$ & $5406^{\mathrm{d}-\mathrm{k}}$ & $4228^{\text {op }}$ & $4783^{\text {ef }}$ \\
\hline & $\mathbf{M}_{3}$ & $5602^{e-n}$ & $6211^{\mathrm{a}-\mathrm{g}}$ & $4801^{\mathrm{n}-\mathrm{t}}$ & $5538^{\text {b-d }}$ & $4403^{e-n}$ & $5054^{\mathrm{c}-\mathrm{i}}$ & $4361^{\mathrm{f}-\mathrm{n}}$ & $4606^{\text {def }}$ & $5003^{g-m}$ & $5633^{\mathrm{d}-\mathrm{h}}$ & $4581^{1-0}$ & $5072^{d e}$ \\
\hline & $\mathbf{M}_{4}$ & $5876^{\mathrm{b}-\mathrm{k}}$ & $6282^{\mathrm{a}-\mathrm{f}}$ & $4969^{1-s}$ & $5709^{\mathrm{bc}}$ & $4987^{\mathrm{d}-\mathrm{k}}$ & $5459^{\mathrm{c}-\mathrm{g}}$ & $4776^{\mathrm{e}-\mathrm{k}}$ & $5074^{\text {cd }}$ & $5432^{\mathrm{d}-\mathrm{k}}$ & $5870^{\mathrm{c}-\mathrm{f}}$ & $4872^{\mathrm{i}-\mathrm{o}}$ & $5391^{\text {cd }}$ \\
\hline \multirow{4}{*}{$\mathbf{S}_{2}$} & $\mathbf{M}_{1}$ & $4400^{q-t}$ & $5933^{b-j}$ & $4107^{t}$ & $4813^{\mathrm{fg}}$ & $4536^{\mathrm{e}-\mathrm{n}}$ & $6221^{\mathrm{a}-\mathrm{c}}$ & $5298^{\mathrm{c}-\mathrm{g}}$ & $\mathbf{5 3 5 2}^{\mathrm{bc}}$ & $4468^{\mathrm{m}-\mathrm{p}}$ & $6077^{\text {b-d }}$ & $4703^{k-0}$ & $5083^{\text {de }}$ \\
\hline & $\mathbf{M}_{2}$ & $5563^{f-0}$ & $6450^{a-e}$ & $5172^{\mathrm{i}-\mathrm{r}}$ & $5728^{\mathrm{bc}}$ & $4630^{e-n}$ & $6616^{\mathrm{ab}}$ & $5235^{\mathrm{c}-\mathrm{h}}$ & $5494^{\mathrm{bc}}$ & $5097^{g-n}$ & $6533^{\mathrm{abc}}$ & $5204^{\mathrm{f}-\mathrm{m}}$ & $5611^{\mathrm{bc}}$ \\
\hline & $\mathbf{M}_{3}$ & $5739^{c-m}$ & $6519^{a-d}$ & $5387^{g-i}$ & $5882^{\text {ab }}$ & $5099^{c-i}$ & $6647^{\mathrm{ab}}$ & $5562^{b-f}$ & $5769^{\mathrm{ab}}$ & $5419^{\mathrm{d}-\mathrm{k}}$ & $6583^{\mathrm{ab}}$ & $5474^{\mathrm{d}-\mathrm{j}}$ & $5825^{\mathrm{ab}}$ \\
\hline & $\mathbf{M}_{4}$ & $6230^{\mathrm{a}-\mathrm{g}}$ & $6838^{a}$ & $5864^{b-t}$ & $6311^{a}$ & $6015^{\mathrm{a}-\mathrm{d}}$ & $6770^{\mathrm{a}}$ & $5589^{\text {b-e }}$ & $6125^{a}$ & $6123^{a-d}$ & $6804^{a}$ & $5727^{\mathrm{d}-\mathrm{g}}$ & $6218^{a}$ \\
\hline \multirow{4}{*}{$\mathbf{S}_{3}$} & $\mathbf{M}_{1}$ & $4696^{0-t}$ & $5264^{i-q}$ & $4244^{\mathrm{st}}$ & $4735^{g}$ & $4339^{g-n}$ & $4558^{\mathrm{e}-\mathrm{n}}$ & $3425^{\mathrm{n}}$ & $4107^{f}$ & $4517^{\mathrm{m}-\mathrm{p}}$ & $4911^{\mathrm{h}-\mathrm{o}}$ & $3834^{\mathrm{p}}$ & $4421^{\mathrm{f}}$ \\
\hline & $\mathbf{M}_{2}$ & $5056^{\mathrm{j}-\mathrm{s}}$ & $6058^{\mathrm{a}-\mathrm{i}}$ & $4873^{\mathrm{m}-\mathrm{t}}$ & $5329^{c-e}$ & $4536^{\mathrm{e}-\mathrm{n}}$ & $4558^{\mathrm{e}-\mathrm{n}}$ & $3484^{\mathrm{mn}}$ & $4193^{f}$ & $4796^{i-0}$ & $5308^{\mathrm{e}-1}$ & $4178^{\text {op }}$ & $4761^{\text {ef }}$ \\
\hline & $\mathbf{M}_{3}$ & $5783^{\mathrm{c}-1}$ & $6584^{\text {abc }}$ & $5047^{\mathrm{k}-\mathrm{s}}$ & $5805^{b}$ & $5261^{\mathrm{chh}}$ & $5414^{\mathrm{c}-\mathrm{g}}$ & $3819^{k-n}$ & $4831^{\text {cde }}$ & $5522^{\mathrm{d}-\mathrm{i}}$ & $6039^{\text {b-e }}$ & $4433^{n-p}$ & $5331^{\text {cd }}$ \\
\hline & $\mathbf{M}_{4}$ & $6119^{a-h}$ & $6725^{\mathrm{ab}}$ & $5185^{\mathrm{i}-\mathrm{q}}$ & $6010^{\mathrm{ab}}$ & $5418^{\mathrm{c}-\mathrm{g}}$ & $6639^{\mathrm{ab}}$ & $4386^{e-n}$ & $5481^{\mathrm{bc}}$ & $5769^{\mathrm{dk}}$ & $6682^{\mathrm{ab}}$ & $4786^{\mathrm{i}-\mathrm{o}}$ & $\mathbf{5 7 3 8}^{\mathrm{bc}}$ \\
\hline \multicolumn{2}{|c|}{ Variety } & $5452^{b}$ & $6191^{\mathrm{a}}$ & $4880^{c}$ & & $4777^{b}$ & $5643^{a}$ & $4446^{c}$ & & $5115^{b}$ & $5917^{\mathrm{a}}$ & $4663^{c}$ & \\
\hline & \multicolumn{4}{|c|}{ Variety $x$ Seed rate } & $\mathbf{S}$ & \multicolumn{3}{|c|}{ Variety $\mathrm{x}$ Seed rate } & $\mathbf{S}$ & \multicolumn{3}{|c|}{ Variety $x$ Seed rate } & $\mathbf{S}$ \\
\hline \multirow{8}{*}{$\mathbf{M}$} & $\mathbf{S}_{1}$ & $5460^{c}$ & $5980^{\mathrm{b}}$ & $4670^{\mathrm{e}}$ & $5370^{b}$ & $4372^{\text {cde }}$ & $5074^{\text {bc }}$ & $4139^{\mathrm{de}}$ & $4528^{b}$ & $4916^{\mathrm{d}}$ & $5527^{\mathrm{bc}}$ & $4405^{\mathrm{e}}$ & $4949^{b}$ \\
\hline & $\mathbf{S}_{2}$ & $5483^{c}$ & $6435^{\mathrm{a}}$ & $5133^{\text {cd }}$ & $5684^{\mathrm{a}}$ & $5070^{\mathrm{bc}}$ & $6564^{\mathrm{a}}$ & $5421^{b}$ & $5685^{\mathrm{a}}$ & $5277^{\text {cd }}$ & $6499^{a}$ & $5277^{\text {cd }}$ & $5684^{\mathrm{a}}$ \\
\hline & $\mathbf{S}_{3}$ & $5414^{\mathrm{c}}$ & $6158^{\mathrm{ab}}$ & $4837^{\mathrm{de}}$ & $5470^{\text {ab }}$ & $4889^{\text {bcd }}$ & $5312^{\mathrm{b}}$ & $3778^{\mathrm{e}}$ & $4660^{b}$ & $5068^{\mathrm{d}}$ & $5735^{\mathrm{b}}$ & $4308^{\mathrm{e}}$ & $5037^{\mathrm{b}}$ \\
\hline & \multicolumn{4}{|c|}{ Variety x Micro } & $\mathbf{M}$ & \multicolumn{3}{|c|}{ Variety x Micro } & $\mathbf{M}$ & \multicolumn{3}{|c|}{ Variety $x$ Micro } & $\mathbf{M}$ \\
\hline & $\mathbf{M}_{1}$ & $4703^{f}$ & $5620^{d}$ & $4218^{g}$ & $4847^{d}$ & $4297^{\text {ef }}$ & $5171^{\text {bcd }}$ & $4097^{\mathrm{f}}$ & $4522^{c}$ & $4500^{\mathrm{fg}}$ & $5396^{\mathrm{b}-\mathrm{d}}$ & $4158^{g}$ & $4685^{c}$ \\
\hline & $\mathbf{M}_{2}$ & $5323^{\mathrm{de}}$ & $6090^{\mathrm{bc}}$ & $4882^{\text {ef }}$ & $5432^{c}$ & $4417^{\mathrm{ef}}$ & $5408^{\mathrm{bc}}$ & $4191^{\mathrm{f}}$ & $4672^{c}$ & $4870^{\text {ef }}$ & $5749^{\mathrm{ab}}$ & $4537^{\mathrm{fg}}$ & 5052 $^{\mathrm{b}}$ \\
\hline & $\mathbf{M}_{3}$ & $5708^{\mathrm{cd}}$ & $6438^{\mathrm{ab}}$ & $5078^{\text {ef }}$ & $5741^{b}$ & $4921^{\text {cde }}$ & $5732^{\mathrm{ab}}$ & $4581^{\text {def }}$ & $\mathbf{5 0 7 8}^{\mathrm{b}}$ & $5315^{\mathrm{cd}}$ & $6085^{\mathrm{a}}$ & $4830^{\text {ef }}$ & $5410^{\mathrm{a}}$ \\
\hline & $\mathbf{M}_{4}$ & $6075^{\mathrm{bc}}$ & $6615^{\mathrm{a}}$ & $5339^{\mathrm{de}}$ & $6010^{a}$ & $5474^{\mathrm{bc}}$ & $6289^{\mathrm{a}}$ & $4917^{\text {cde }}$ & $5560^{a}$ & $5663^{\mathrm{a}-\mathrm{c}}$ & $6052^{a}$ & $5128^{\mathrm{de}}$ & $5614^{\mathrm{a}}$ \\
\hline \multicolumn{2}{|c|}{ Comparison } & \multicolumn{4}{|c|}{ S.Em \pm} & \multicolumn{4}{|c|}{ S.Em \pm} & \multicolumn{4}{|c|}{ S.Em \pm} \\
\hline \multicolumn{2}{|c|}{ Variety (V) } & \multicolumn{4}{|c|}{57.6} & \multicolumn{4}{|c|}{75.2} & \multicolumn{4}{|c|}{55.7} \\
\hline \multicolumn{2}{|c|}{ Seed (S) } & \multicolumn{4}{|c|}{69.6} & \multicolumn{4}{|c|}{157.8} & & 68 & & \\
\hline Mi & (M) & & & & & & & & & & 74 & & \\
\hline & & & & & & & & & & & 11 & & \\
\hline & & & & & & & & & & & 12 & & \\
\hline & & & & & & & & & & & 12 & & \\
\hline$V_{x}$ & & & & & & & & & & & 22 & & \\
\hline
\end{tabular}

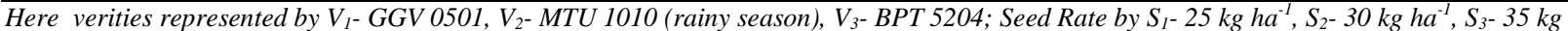

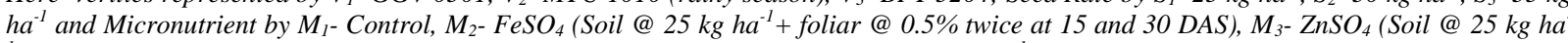
+ foliar @ 0.5\% twice at 15 and $30 \mathrm{DAS}$ ), $\mathrm{M}_{4-} \mathrm{FeSO}_{4}+\mathrm{ZnSO}_{4}$ (each to soil @ $25 \mathrm{~kg} \mathrm{ha} \mathrm{l}^{-1}$ foliar @ 0.5\% at 15 and $30 \mathrm{DAS}$ ), The values between the same set of classes for each treatment followed by the same letter are not significantly different under DMRT

$\mathrm{M}_{4}-\mathrm{M}_{2}+\mathrm{M}_{3}$. Normal crop husbandry practices were followed for successful rising of the crop. The statistical analysis were carried out to obtained data and tested at five per cent level of significance to interpret the treatment difference (Gomez \& Gomez, 2010).

Overall, variations in pooled data were highlighted unless otherwise individual years behaved differently. In the present investigation during rainy season, cv. MTU 1010 recorded significantly higher grain yield $\left(5917 \mathrm{~kg} \mathrm{ha}^{-1}\right)$ over cultivars GGV $0501\left(5115 \mathrm{~kg} \mathrm{ha}^{-1}\right)$ and BPT $5204\left(4663 \mathrm{~kg} \mathrm{ha}^{-1}\right)$. The increase in yield with cv. MTU 1010 was to the tune of 15.6 and $26.9 \%$

respectively over GGV 0501 and BPT 5204 cultivars (Table 2). The coarse grain cultivar, MTU 1010 is found suitable for DSR 
Table 3 Effect of genotypes, seed rates and micronutrients on net returns $\left(\mathrm{Cha}^{-1}\right)$ under direct seeded condition during rainy season

\begin{tabular}{|c|c|c|c|c|c|c|c|c|c|c|c|c|c|}
\hline \multirow{2}{*}{\multicolumn{2}{|c|}{$\mathrm{V} \times \mathrm{S} \times \mathrm{M}$}} & \multicolumn{4}{|c|}{ 2014-15 } & \multicolumn{4}{|c|}{$2015-16$} & \multicolumn{4}{|c|}{ Pooled } \\
\hline & & $\mathrm{V}_{1}$ & $\mathrm{~V}_{2}$ & $\mathrm{~V}_{3}$ & $\mathrm{~S} \times \mathrm{M}$ & $\mathrm{V}_{1}$ & $\mathrm{~V}_{2}$ & $\mathrm{~V}_{3}$ & $\mathrm{~S} \times \mathrm{M}$ & $\mathrm{V}_{1}$ & $\mathrm{~V}_{2}$ & $\mathrm{~V}_{3}$ & S x M \\
\hline \multirow{4}{*}{$\mathrm{S}_{1}$} & $\mathrm{M}_{1}$ & $50939^{\mathrm{g}-\mathrm{k}}$ & $53088^{\mathrm{f}-\mathrm{k}}$ & $44144^{\mathrm{i}-\mathrm{k}}$ & $49390^{\text {eg }}$ & $34879^{\mathrm{a}}$ & $41549^{\mathrm{a}}$ & $33110^{\mathrm{a}}$ & $36513^{b}$ & $\underset{\mathrm{m}}{43293^{\mathrm{k}-}}$ & $\underset{n}{47678^{\text {h- }}}$ & $37915^{\mathrm{mn}}$ & $42962^{f}$ \\
\hline & $\mathrm{M}_{2}$ & $56437^{\mathrm{e}-\mathrm{i}}$ & $54304^{\mathrm{f}-\mathrm{j}}$ & $49542^{\mathrm{h}-\mathrm{k}}$ & $53428^{\mathrm{d}-\mathrm{f}}$ & $35614^{\mathrm{a}}$ & $47277^{\mathrm{a}}$ & $39411^{\mathrm{a}}$ & $40767^{\mathrm{ab}}$ & $45633^{\mathrm{j}-\mathrm{n}}$ & $51465^{\mathrm{f}-\mathrm{l}}$ & $44057^{\mathrm{j}-\mathrm{n}}$ & $47051^{\mathrm{ef}}$ \\
\hline & $\mathrm{M}_{3}$ & $61614^{\mathrm{b}-\mathrm{h}}$ & $64719^{a-g}$ & $54082^{\mathrm{f}-\mathrm{j}}$ & $60138^{\mathrm{cd}}$ & $41603^{a}$ & $47616^{\mathrm{a}}$ & $50286^{\mathrm{a}}$ & $46502^{\mathrm{ab}}$ & $51286^{\mathrm{f}-\mathrm{l}}$ & $\underset{\mathrm{k}}{56292^{\mathrm{e}-}}$ & $51984^{\mathrm{e}-1}$ & $53187^{\mathrm{de}}$ \\
\hline & $\mathrm{M}_{4}$ & $66612^{\mathrm{a}-\mathrm{f}}$ & $65188^{\mathrm{a}-\mathrm{g}}$ & $56709^{\mathrm{e}-\mathrm{i}}$ & $62836^{\mathrm{bc}}$ & $52851^{\mathrm{a}}$ & $54924^{\mathrm{a}}$ & $58478^{\mathrm{a}}$ & $55417^{\mathrm{ab}}$ & $59402^{\mathrm{d}-\mathrm{i}}$ & $59766^{\mathrm{d}-\mathrm{i}}$ & $57136^{\mathrm{e}-1}$ & $58768^{\mathrm{cd}}$ \\
\hline \multirow{4}{*}{$\mathrm{S}_{2}$} & $\mathrm{M}_{1}$ & $40503^{j-k}$ & $60635^{\mathrm{c}-\mathrm{h}}$ & $39574^{\mathrm{k}}$ & $46904^{f-g}$ & $46376^{\mathrm{a}}$ & $70455^{\mathrm{a}}$ & $71142^{\mathrm{a}}$ & $62658^{\mathrm{ab}}$ & $42331^{1-m}$ & $65269^{\mathrm{a}-\mathrm{e}}$ & $54815^{\mathrm{e}-1}$ & $54138^{d}$ \\
\hline & $\mathrm{M}_{2}$ & $61112^{\mathrm{b}-\mathrm{h}}$ & $68731^{\mathrm{a}-\mathrm{e}}$ & $60479^{\mathrm{c}-\mathrm{h}}$ & $63441^{\mathrm{bc}}$ & $47533^{a}$ & $77966^{\mathrm{a}}$ & $70326^{\mathrm{a}}$ & $65275^{\mathrm{ab}}$ & $53918^{\mathrm{e}-1}$ & $73050^{\mathrm{a}-\mathrm{c}}$ & $65275^{a-e}$ & $64081^{\mathrm{bc}}$ \\
\hline & $\mathrm{M}_{3}$ & $65198^{\mathrm{a}-\mathrm{g}}$ & $71448^{\mathrm{a}-\mathrm{d}}$ & $66591^{\mathrm{a}-\mathrm{f}}$ & $67746^{\mathrm{b}}$ & $57405^{a}$ & $78859^{a}$ & $77655^{a}$ & $71307^{\mathrm{ab}}$ & $60774^{\mathrm{ch} h}$ & $74674^{\mathrm{ab}}$ & $71410^{\mathrm{a}-\mathrm{d}}$ & $68953^{b}$ \\
\hline & $\mathrm{M}_{4}$ & $73658^{a-c}$ & $77680^{\mathrm{a}}$ & $75270^{\mathrm{ab}}$ & $75536^{a}$ & $74959^{\mathrm{a}}$ & $80510^{\mathrm{a}}$ & $78036^{a}$ & $77835^{\mathrm{a}}$ & $73828^{\mathrm{ab}}$ & $78084^{a}$ & $76533^{a}$ & $76148^{a}$ \\
\hline \multirow{4}{*}{$\mathrm{S}_{3}$} & $\mathrm{M}_{1}$ & $44321^{\mathrm{i}-\mathrm{k}}$ & $47846^{\mathrm{h}-\mathrm{k}}$ & $42047^{\mathrm{jk}}$ & $44738^{g}$ & $42570^{\mathrm{a}}$ & $37928^{a}$ & $29160^{\mathrm{a}}$ & $36553^{b}$ & $42782^{1-m}$ & $\underset{\mathrm{n}}{43048^{\mathrm{k}-}}$ & $35394^{n}$ & $40408^{f}$ \\
\hline & $\mathrm{M}_{2}$ & $51211^{\mathrm{g}-\mathrm{k}}$ & $61633^{b-h}$ & $54194^{\mathrm{f}-\mathrm{j}}$ & $55679^{\mathrm{de}}$ & $45940^{\mathrm{a}}$ & $37319^{a}$ & $29695^{\mathrm{a}}$ & $37651^{b}$ & $48081^{\mathrm{h}-\mathrm{n}}$ & $49247^{\mathrm{g}-\mathrm{m}}$ & $41592^{1-n}$ & $46307^{f}$ \\
\hline & $\mathrm{M}_{3}$ & $65619^{\mathrm{a}-\mathrm{f}}$ & $71256^{\mathrm{a}-\mathrm{d}}$ & $58145^{\mathrm{d}-\mathrm{i}}$ & $65007^{\mathrm{bc}}$ & $60532^{a}$ & $55544^{\mathrm{a}}$ & $37507^{\mathrm{a}}$ & $51194^{\mathrm{ab}}$ & $62449^{\mathrm{b}-\mathrm{g}}$ & $63154^{\text {b-f }}$ & $47302^{i-n}$ & $57635^{\mathrm{cd}}$ \\
\hline & $\mathrm{M}_{4}$ & $71252^{a-d}$ & $72656^{\mathrm{a}-\mathrm{c}}$ & $61303^{\mathrm{b}-\mathrm{h}}$ & $68404^{b}$ & $62966^{\mathrm{a}}$ & $76998^{a}$ & $50451^{\mathrm{a}}$ & $63472^{\mathrm{ab}}$ & $59349^{\mathrm{d}-\mathrm{i}}$ & $75092^{\mathrm{ab}}$ & $55038^{\mathrm{e}-\mathrm{l}}$ & $63160^{\text {bc }}$ \\
\hline \multicolumn{2}{|c|}{ Variety } & $59040^{\mathrm{b}}$ & $64099^{a}$ & $55173^{\mathrm{b}}$ & & $50269^{\mathrm{b}}$ & $58912^{\mathrm{a}}$ & $52105^{\mathrm{b}}$ & & $53594^{\mathrm{b}}$ & $61402^{\mathrm{a}}$ & $53204^{\mathrm{b}}$ & \\
\hline & \multicolumn{4}{|c|}{ Variety $x$ Seed rate } & $S$ & \multicolumn{3}{|c|}{ Variety $x$ Seed rate } & $S$ & \multicolumn{3}{|c|}{ Variety $x$ Seed rate } & $S$ \\
\hline \multirow{4}{*}{$\mathrm{S}$} & $\mathrm{S}_{1}$ & $58900^{\mathrm{bc}}$ & $59325^{\mathrm{bc}}$ & $51119^{d}$ & $56448^{b}$ & $41237^{\mathrm{a}}$ & $47841^{\mathrm{a}}$ & $45321^{\mathrm{a}}$ & $44800^{\mathrm{b}}$ & $49903^{e}$ & $53800^{d}$ & $47773^{f}$ & $50492^{c}$ \\
\hline & $\mathrm{S}_{2}$ & $60118^{\mathrm{bc}}$ & $69623^{\mathrm{a}}$ & $60479^{\mathrm{bc}}$ & $63407^{\mathrm{a}}$ & $56568^{\mathrm{a}}$ & $76947^{\mathrm{a}}$ & $74290^{\mathrm{a}}$ & $69269^{\mathrm{a}}$ & $57713^{c}$ & $72769^{a}$ & $67008^{b}$ & $65830^{\mathrm{a}}$ \\
\hline & $\mathrm{S}_{3}$ & $58101^{\mathrm{b}-\mathrm{d}}$ & $63348^{\mathrm{ab}}$ & $53922^{\mathrm{cd}}$ & $58457^{\mathrm{b}}$ & $45321^{\mathrm{a}}$ & $74290^{a}$ & $36703^{a}$ & $47217^{\mathrm{b}}$ & $53165^{d}$ & $57635^{\mathrm{c}}$ & $44831^{g}$ & $51877^{\mathrm{b}}$ \\
\hline & \multicolumn{4}{|c|}{ Variety x Micro } & M & \multicolumn{3}{|c|}{ Variety x Micro } & M & \multicolumn{3}{|c|}{ Variety x Micro } & M \\
\hline \multirow{4}{*}{ M } & $\mathrm{M}_{1}$ & $45254^{f}$ & $53856^{\mathrm{e}}$ & $41922^{f}$ & $47011^{d}$ & $41275^{\mathrm{a}}$ & $49977^{\mathrm{a}}$ & $44471^{\mathrm{a}}$ & $45241^{\mathrm{d}}$ & $42802^{g}$ & $51998^{\mathrm{d}-\mathrm{f}}$ & $42708^{\mathrm{g}}$ & $45836^{\mathrm{d}}$ \\
\hline & $\mathrm{M}_{2}$ & $56253^{\mathrm{de}}$ & $61556^{\mathrm{cd}}$ & $54738^{\mathrm{de}}$ & $57516^{\mathrm{c}}$ & $43029^{a}$ & $54187^{\mathrm{a}}$ & $46477^{\mathrm{a}}$ & $47898^{c}$ & $49211^{\mathrm{f}}$ & $57921^{\mathrm{b}-\mathrm{d}}$ & $50308^{\mathrm{ef}}$ & $52480^{\mathrm{c}}$ \\
\hline & $\mathrm{M}_{3}$ & $64144^{\mathrm{bc}}$ & $69141^{\mathrm{ab}}$ & $59606^{\mathrm{c}-\mathrm{e}}$ & $64297^{\mathrm{b}}$ & $53180^{\mathrm{a}}$ & $60673^{a}$ & $55149^{\mathrm{a}}$ & $56334^{\mathrm{b}}$ & $58169^{\mathrm{b}-\mathrm{d}}$ & $64707^{\mathrm{ab}}$ & $\begin{array}{c}56899 \mathrm{c}- \\
\mathrm{e}\end{array}$ & $59925^{b}$ \\
\hline & $\mathrm{M}_{4}$ & $70507^{\mathrm{ab}}$ & $71841^{\mathrm{a}}$ & $64427^{\mathrm{bc}}$ & $68925^{a}$ & $63592^{\mathrm{a}}$ & $70810^{a}$ & $62321^{\mathrm{a}}$ & $65575^{\mathrm{a}}$ & $64193^{b}$ & $70981^{a}$ & $62902^{\mathrm{bc}}$ & $66025^{a}$ \\
\hline \multicolumn{2}{|c|}{ Comparison } & \multicolumn{4}{|c|}{ S.Em \pm} & \multicolumn{4}{|c|}{ S.Em \pm} & \multicolumn{4}{|c|}{ S.Em \pm} \\
\hline \multicolumn{2}{|c|}{ Variety (V) } & \multicolumn{4}{|c|}{1198} & \multicolumn{4}{|c|}{1361} & \multicolumn{4}{|c|}{1089} \\
\hline \multicolumn{2}{|c|}{ Seed $(S)$} & \multicolumn{4}{|c|}{1262} & & & & & & 12 & & \\
\hline $\mathrm{Mi}$ & & & & & & & & & & & 130 & & \\
\hline & & & & 85 & & & & & & & 213 & & \\
\hline & & & & 95 & & & 37 & & & & 22 & & \\
\hline & & & & 95 & & & & & & & 22 & & \\
\hline$V_{x}$ & & & & & & & & & & & 39 & & \\
\hline
\end{tabular}

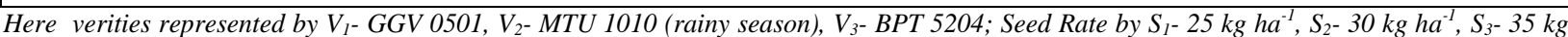

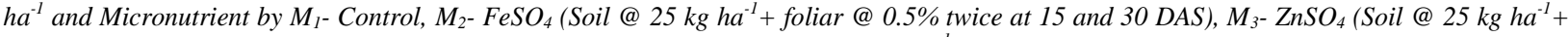

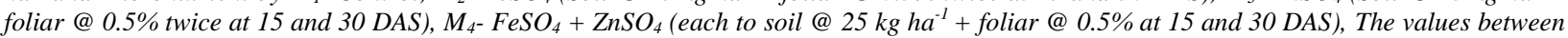
the same set of classes for each treatment followed by the same letter are not significantly different under DMRT 
Table 4 Effect of genotypes, seed rates and micronutrients on B: C under direct seeded condition during rainy season

\begin{tabular}{|c|c|c|c|c|c|c|c|c|c|c|c|c|c|}
\hline \multirow{2}{*}{\multicolumn{2}{|c|}{ V x S x M }} & \multicolumn{4}{|c|}{ 2014-15 } & \multicolumn{4}{|c|}{$2015-16$} & \multicolumn{4}{|c|}{ Pooled } \\
\hline & & $\mathrm{V}_{1}$ & $\mathrm{~V}_{2}$ & $\mathrm{~V}_{3}$ & $\mathrm{~S} \times \mathrm{M}$ & $\mathrm{V}_{1}$ & $\mathrm{~V}_{2}$ & $\mathrm{~V}_{3}$ & $\mathrm{~S} \times \mathrm{M}$ & $\mathrm{V}_{1}$ & $\mathrm{~V}_{2}$ & $\mathrm{~V}_{3}$ & $\mathrm{~S} \times \mathrm{M}$ \\
\hline \multirow{4}{*}{$\mathrm{S}_{1}$} & $\mathrm{M}_{1}$ & $2.00^{\mathrm{e}-\mathrm{j}}$ & $2.04^{\mathrm{d}-\mathrm{j}}$ & $1.86^{\mathrm{h}-\mathrm{j}}$ & $1.97^{\mathrm{ef}}$ & $1.67^{\mathrm{i}-\mathrm{k}}$ & $1.80^{\mathrm{h}-\mathrm{k}}$ & $1.64^{\mathrm{jk}}$ & $1.70^{\mathrm{f}}$ & $1.84^{\mathrm{l}-\mathrm{p}}$ & $1.93^{\mathrm{i}-\mathrm{p}}$ & $1.73^{\mathrm{op}}$ & $1.83^{\mathrm{g}}$ \\
\hline & $\mathrm{M}_{2}$ & $2.09^{c-j}$ & $2.05^{\mathrm{d}-\mathrm{j}}$ & $1.95^{\mathrm{f}-\mathrm{j}}$ & $2.03^{\mathrm{d}-\mathrm{f}}$ & $1.67^{\mathrm{i}-\mathrm{k}}$ & $1.90^{\mathrm{h}-\mathrm{k}}$ & $1.75^{\mathrm{h}-\mathrm{k}}$ & $1.77^{\mathrm{ef}}$ & $1.87^{\mathrm{k}-\mathrm{p}}$ & $1.99^{\mathrm{f}-\mathrm{n}}$ & $1.84^{\mathrm{l}-\mathrm{p}}$ & $1.90^{\mathrm{fg}}$ \\
\hline & $\mathrm{M}_{3}$ & $2.19^{\mathrm{a}-\mathrm{g}}$ & $2.26^{\mathrm{a}-\mathrm{f}}$ & $2.04^{\mathrm{d}-\mathrm{j}}$ & $2.16^{\mathrm{b}-\mathrm{d}}$ & $1.79^{\mathrm{h}-\mathrm{k}}$ & $1.91^{\mathrm{h}-\mathrm{k}}$ & $1.95^{\mathrm{f}-\mathrm{k}}$ & $1.88^{\mathrm{d}-\mathrm{f}}$ & $1.98^{\mathrm{g}-\mathrm{o}}$ & $2.08^{\mathrm{e}-1}$ & $1.99^{\mathrm{f}-\mathrm{n}}$ & $2.02^{\mathrm{ef}}$ \\
\hline & $\mathrm{M}_{4}$ & $2.26^{\mathrm{a}-\mathrm{f}}$ & $2.24^{\mathrm{a}-\mathrm{g}}$ & $2.08^{\mathrm{c}-\mathrm{j}}$ & $2.19^{b-d}$ & $1.98^{e-j}$ & $2.03^{\mathrm{d}-\mathrm{j}}$ & $2.09^{\mathrm{a}-\mathrm{i}}$ & $2.03^{\mathrm{cd}}$ & $2.12^{\mathrm{d}-\mathrm{k}}$ & $2.13^{\mathrm{d}-\mathrm{j}}$ & $2.07^{\mathrm{e}-\mathrm{m}}$ & $2.11^{\mathrm{c}-\mathrm{e}}$ \\
\hline \multirow{4}{*}{$\mathrm{S}_{2}$} & $\mathrm{M}_{1}$ & $1.79^{\mathrm{j}}$ & $2.19^{\mathrm{a}-\mathrm{g}}$ & $1.77^{\mathrm{j}}$ & $1.92^{\mathrm{ef}}$ & $1.89^{\mathrm{h}-\mathrm{k}}$ & $2.35^{\mathrm{a}-\mathrm{f}}$ & $2.36^{\mathrm{a}-\mathrm{e}}$ & $2.20^{\mathrm{bc}}$ & $1.82^{\mathrm{m}-\mathrm{p}}$ & $2.27^{\mathrm{a}-\mathrm{e}}$ & $2.06^{\mathrm{e}-\mathrm{m}}$ & $2.05^{\mathrm{de}}$ \\
\hline & $\mathrm{M}_{2}$ & $2.17^{\mathrm{a}-\mathrm{h}}$ & $2.33^{\mathrm{a}-\mathrm{d}}$ & $2.16^{\mathrm{a}-\mathrm{h}}$ & $2.22^{\mathrm{bc}}$ & $1.90^{\mathrm{h}-\mathrm{k}}$ & $2.48^{\mathrm{ab}}$ & $2.33^{a-g}$ & $2.23^{\mathrm{bc}}$ & $2.03^{\mathrm{e}-\mathrm{n}}$ & $2.40^{\mathrm{a}-\mathrm{c}}$ & $2.24^{\mathrm{a}-\mathrm{f}}$ & $2.22^{\mathrm{bc}}$ \\
\hline & $\mathrm{M}_{3}$ & $2.26^{\mathrm{a}-\mathrm{f}}$ & $2.38^{\mathrm{ac}-\mathrm{c}}$ & $2.28^{\mathrm{ae}}$ & $2.31^{\mathrm{ab}}$ & $2.08^{b-i}$ & $2.50^{\mathrm{a}}$ & $2.47^{\mathrm{ab}}$ & $2.35^{\mathrm{ab}}$ & $2.16^{1-\mathrm{i}}$ & $2.43^{\mathrm{ab}}$ & $2.36^{\mathrm{a}-\mathrm{d}}$ & $2.32^{\mathrm{ab}}$ \\
\hline & $\mathrm{M}_{4}$ & $2.39^{a-c}$ & $2.48^{\mathrm{a}}$ & $2.42^{\mathrm{ab}}$ & $2.43^{\mathrm{a}}$ & $2.39^{\mathrm{a}-\mathrm{d}}$ & $2.50^{\mathrm{a}}$ & $2.45^{a-c}$ & $2.45^{\mathrm{a}}$ & $2.38^{\mathrm{a}-\mathrm{c}}$ & $2.47^{\mathrm{a}}$ & $2.43^{\text {ad }}$ & $2.43^{\mathrm{a}}$ \\
\hline \multirow{4}{*}{$\mathrm{S}_{3}$} & $\mathrm{M}_{1}$ & $1.86^{\mathrm{h}-\mathrm{j}}$ & $1.93^{\mathrm{g}-\mathrm{j}}$ & $1.82^{\mathrm{ij}}$ & $1.87^{\mathrm{f}}$ & $1.81^{\mathrm{h}-\mathrm{k}}$ & $1.73^{\mathrm{i}-\mathrm{k}}$ & $1.56^{\mathrm{k}}$ & $1.70^{\mathrm{f}}$ & $1.82^{\mathrm{m}-\mathrm{p}}$ & $1.83^{1-p}$ & $1.68^{\mathrm{p}}$ & $1.78^{\mathrm{g}}$ \\
\hline & $\mathrm{M}_{2}$ & $1.98^{e-j}$ & $2.19^{\mathrm{a}-\mathrm{g}}$ & $2.04^{\mathrm{d}-\mathrm{j}}$ & $2.07^{\mathrm{c}-\mathrm{e}}$ & $1.86^{\mathrm{h}-\mathrm{k}}$ & $1.70^{\mathrm{i}-\mathrm{k}}$ & $1.56^{\mathrm{k}}$ & $1.71^{\mathrm{f}}$ & $1.91^{\mathrm{i}-\mathrm{p}}$ & $1.94^{\mathrm{h}-\mathrm{o}}$ & $1.79^{\mathrm{n}-\mathrm{p}}$ & $1.88^{\mathrm{g}}$ \\
\hline & $\mathrm{M}_{3}$ & $2.26^{\mathrm{a}-\mathrm{f}}$ & $2.38^{a-c}$ & $2.12^{b-i}$ & $2.25^{\mathrm{b}}$ & $2.4^{\mathrm{a}-\mathrm{d}}$ & $2.05^{c-j}$ & $1.70^{\mathrm{i}-\mathrm{k}}$ & $1.97^{\mathrm{de}}$ & $2.19^{\mathrm{b}-\mathrm{h}}$ & $2.21^{\mathrm{b}-\mathrm{g}}$ & $1.90^{j-p}$ & $2.10^{\mathrm{c}-\mathrm{e}}$ \\
\hline & $\mathrm{M}_{4}$ & $2.34^{\mathrm{a}-\mathrm{d}}$ & $2.38^{\mathrm{a}-\mathrm{c}}$ & $2.15^{\mathrm{b}-\mathrm{h}}$ & $2.29^{\mathrm{ab}}$ & $2.16^{\mathrm{a}-\mathrm{h}}$ & $2.43^{\mathrm{a}-\mathrm{d}}$ & $1.93^{\mathrm{g}-\mathrm{k}}$ & $2.18^{\mathrm{bc}}$ & $2.11^{\mathrm{e}-\mathrm{k}}$ & $2.41^{\mathrm{ab}}$ & $2.03^{e-n}$ & $2.18^{\mathrm{cd}}$ \\
\hline \multicolumn{2}{|c|}{ Variety } & $2.13^{\mathrm{b}}$ & $2.24^{\mathrm{a}}$ & $2.06^{\mathrm{b}}$ & & $1.95^{\mathrm{b}}$ & $2.11^{\mathrm{a}}$ & $1.98^{\mathrm{b}}$ & & $2.02^{\mathrm{b}}$ & $2.17^{\mathrm{a}}$ & $2.01^{\mathrm{b}}$ & \\
\hline & \multicolumn{4}{|c|}{ Variety $x$ Seed rate } & S & \multicolumn{3}{|c|}{ Variety $x$ Seed rate } & S & \multicolumn{3}{|c|}{ Variety x Seed rate } & S \\
\hline \multirow{4}{*}{ S } & $\mathrm{S}_{1}$ & $2.13^{\mathrm{bc}}$ & $2.15^{\mathrm{bc}}$ & $1.98^{\mathrm{d}}$ & $2.09^{\mathrm{b}}$ & $1.78^{\mathrm{bc}}$ & $1.91^{\mathrm{bc}}$ & $1.86^{\mathrm{b}}$ & $1.85^{\mathrm{b}}$ & $1.95^{\mathrm{cd}}$ & $2.03^{\mathrm{bc}}$ & $1.91^{\mathrm{cd}}$ & $1.96^{\mathrm{b}}$ \\
\hline & $\mathrm{S}_{2}$ & $2.15^{\mathrm{bc}}$ & $2.34^{\mathrm{a}}$ & $2.16^{\mathrm{bc}}$ & $2.22^{\mathrm{a}}$ & $2.06^{\mathrm{b}}$ & $2.46^{\mathrm{a}}$ & $2.40^{\mathrm{a}}$ & $2.31^{\mathrm{a}}$ & $2.10^{\mathrm{b}}$ & $2.39^{\mathrm{a}}$ & $2.27^{\mathrm{a}}$ & $2.25^{\mathrm{a}}$ \\
\hline & $\mathrm{S}_{3}$ & $2.11^{\mathrm{b}-\mathrm{d}}$ & $2.22^{\mathrm{ab}}$ & $2.03^{\mathrm{cd}}$ & $2.12^{\mathrm{b}}$ & $1.99^{\mathrm{bc}}$ & $1.98^{\mathrm{bc}}$ & $1.69^{\mathrm{c}}$ & $1.89^{\mathrm{b}}$ & $2.01^{\mathrm{bc}}$ & $2.10^{\mathrm{b}}$ & $1.85^{\mathrm{d}}$ & $1.98^{\mathrm{b}}$ \\
\hline & \multicolumn{4}{|c|}{ Variety x Micro } & M & \multicolumn{3}{|c|}{ Variety x Micro } & M & \multicolumn{3}{|c|}{ Variety x Micro } & M \\
\hline \multirow{4}{*}{ M } & $\mathrm{M}_{1}$ & $1.88^{\mathrm{e}}$ & $2.06^{\mathrm{cd}}$ & $1.82^{\mathrm{e}}$ & $1.92^{\mathrm{c}}$ & $1.79^{\mathrm{e}}$ & $1.96^{\mathrm{be}}$ & $1.85^{\mathrm{ce}}$ & $1.87^{\mathrm{c}}$ & $1.83^{\mathrm{f}}$ & $2.01^{\mathrm{de}}$ & $1.82^{\mathrm{f}}$ & $1.89^{\mathrm{d}}$ \\
\hline & $\mathrm{M}_{2}$ & $2.08^{\mathrm{cd}}$ & $2.19^{\mathrm{b}-\mathrm{d}}$ & $2.05^{\mathrm{d}}$ & $2.11^{\mathrm{b}}$ & $1.81^{\mathrm{de}}$ & $2.03^{b-d}$ & $1.88^{\mathrm{c}-\mathrm{e}}$ & $1.90^{\mathrm{c}}$ & $1.94^{\mathrm{ef}}$ & $2.11^{b-d}$ & $1.96^{\mathrm{e}}$ & $2.00^{\mathrm{c}}$ \\
\hline & $\mathrm{M}_{3}$ & $2.23^{\mathrm{a}-\mathrm{c}}$ & $2.34^{\mathrm{ab}}$ & $2.15^{\mathrm{cd}}$ & $2.24^{\mathrm{a}}$ & $2.00^{\mathrm{b}-\mathrm{e}}$ & $2.15^{\mathrm{ab}}$ & $2.04^{\mathrm{bc}}$ & $2.07^{\mathrm{b}}$ & $2.11^{\mathrm{b}-\mathrm{d}}$ & $2.24^{\mathrm{ab}}$ & $2.09^{\mathrm{cd}}$ & $2.14^{\mathrm{b}}$ \\
\hline & $\mathrm{M}_{4}$ & $2.33^{\mathrm{ab}}$ & $2.37^{\mathrm{a}}$ & $2.22^{\mathrm{a}-\mathrm{d}}$ & $2.31^{\mathrm{a}}$ & $2.18^{\mathrm{ab}}$ & $2.32^{\mathrm{a}}$ & $2.16^{\mathrm{ab}}$ & $2.22^{\mathrm{a}}$ & $2.20^{\mathrm{bc}}$ & $2.34^{\mathrm{a}}$ & $2.18^{\mathrm{bc}}$ & $2.24^{\mathrm{a}}$ \\
\hline \multicolumn{2}{|c|}{ Comparison } & \multicolumn{4}{|c|}{ S.Em \pm} & \multicolumn{4}{|c|}{ S.Em \pm} & \multicolumn{4}{|c|}{ S.Em \pm} \\
\hline \multicolumn{2}{|c|}{ Variety (V) } & \multicolumn{4}{|c|}{0.02} & \multicolumn{4}{|c|}{0.03} & \multicolumn{4}{|c|}{0.02} \\
\hline \multicolumn{2}{|c|}{ Seed (S) } & \multicolumn{4}{|c|}{0.02} & & & & & & & & \\
\hline Micro & & & & & & & & & & & & & \\
\hline $\mathrm{V} \times \mathrm{S}$ & & & & & & & & & & & & & \\
\hline $\mathrm{V} \times \mathrm{N}$ & & & & & & & & & & & & & \\
\hline $\mathrm{S} \times \mathrm{M}$ & & & & & & & & & & & & & \\
\hline $\mathrm{V} \times \mathrm{S}$ & & & & & & & & & & & & & \\
\hline
\end{tabular}

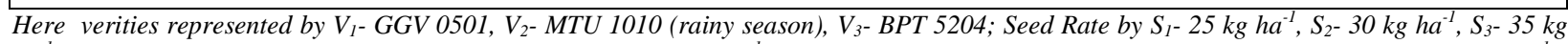

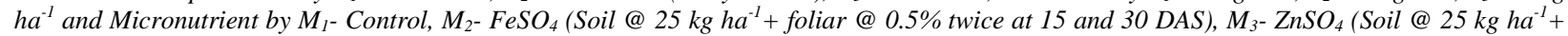
foliar@0.5\% twice at 15 and $30 \mathrm{DAS}$ ), $\mathrm{M}_{4^{-}} \mathrm{FeSO}_{4}+\mathrm{ZnSO}_{4}$ (each to soil @ $25 \mathrm{~kg} \mathrm{ha}{ }^{-1}+$ foliar @ $0.5 \%$ at 15 and $30 \mathrm{DAS}$ ), The values between the same set of classes for each treatment followed by the same letter are not significantly different under DMRT 
ecosystem with higher yield potential. MTU 1010 being early 10 15 days it could be preferred over others under moisture constraint situations to make best use of the scarce moisture. Similar results were obtained by Zhao et al. (2007), Awan et al. (2011) and Yadav et al. (2017) in aerobic rice under different rice ecosystems. The finding draws its strength from Kiran et al. (2015) who also worked in the TBP irrigation command earlier and reported superior performance of cv. JKPH 3333, followed by GGV 0501 and BPT 5204 cultivars.

Among seed rates, a medium seed rate of $30 \mathrm{~kg} \mathrm{ha}^{-1}$ across cultivars recorded higher grain $\left(5684 \mathrm{~kg} \mathrm{ha}^{-1}\right.$ ) (Table 2). Better performance of DSR with moderate seed rate in terms of seed yield and straw yield due to better performance of growth and yield components under moderate seed rate. In the Indo-Gangetic plain, in India also a seed rate of 20-25 kg ha ${ }^{-1}$ was found optimum for medium fine grain rice cultivars (Gopal et al., 2010). Application of $\mathrm{FeSO}_{4}$ and $\mathrm{ZnSO}_{4}$ both basally to the soil and to the foliage subsequently recorded higher grain yield (5614 $\mathrm{kg} \mathrm{ha}^{-1}$ ). At Parbhani, similar to present finding Jadhav et al. (2014) obtained significantly higher yields with combined application of $\mathrm{FeSO}_{4}$ and $\mathrm{ZnSO}_{4}$ at $10 \mathrm{~kg} \mathrm{ha}^{-1}$ each. In Over all interactions, cv. MTU 1010 sown with $30 \mathrm{~kg} \mathrm{ha}^{-1}$ seed rate and applied with both $\mathrm{FeSO}_{4}$ and $\mathrm{ZnSO}_{4}$ besides recommended NPK (150:75:75 $\left.\mathrm{kg} \mathrm{ha}^{-1}\right)$ recorded higher grain yield $\left(6804 \mathrm{~kg} \mathrm{ha}^{-1}\right.$ ) (Table 2). Yadav et al. (2017) obtained higher yield of hybrid cultivars DRRH-3 and PAC 837 with higher seed rate of $35 \mathrm{~kg} \mathrm{ha}^{-1}$ under aerobic cultivation, and it being coarse cultivar with bold grains higher seed rate could be expected unlike other cultivars used in present study. As far micronutrient nutrition is concerned, Gill et al. (2006) obtained higher yield with foliar nutrition of $\mathrm{MnSO}_{4}$ and $\mathrm{FeSO}_{4}$ with Basmati rice at Ludhiana.

Net returns of Rs. 78,084 was higher with cv. MTU 1010 sown using $30 \mathrm{~kg} \mathrm{ha}^{-1}$ and applied with both $\mathrm{FeSO}_{4}$ and $\mathrm{ZnSO}_{4}$ followed by cv. BPT $5204\left(\right.$ Rs.76,533 ha $\left.{ }^{-1}\right)$ at $30 \mathrm{~kg} \mathrm{ha}^{-1}$ and applied with both $\mathrm{FeSO}_{4}$ and $\mathrm{ZnSO}_{4}$ (Table 3). Similar trend was obtained in Benefit cost ratio of 2.47 and 2.43 (Table 4).

\section{Conflict of Interest}

Authors would hereby like to declare that there is no conflict of interests that could possibly arise.

\section{References}

Awan TH, Ali RI, Manzoor Z, Ahmad M, Akhtar M (2011) Effect of different nitrogen levels and row spacing on the performance of newly evolved medium grain rice variety, KSK-133. Journal of Anima land Plant Science 21: 231-234.

Food and Agriculture Organization (2014) Rice and Us. Available on http://www.fao.org/rice2014/en/aboutrice.html access on 25January, 2018.
Food and Agriculture Organization (2017) The future of food and agriculture- trends and challenges. Available on http://www.fao.org/publication.html access on 25January, 2018.

Gill MS, Kumar P, Kumar A (2006) Growth and yield of directseeded rice (Oryza sativa) as influenced by seeding technique and seed rate under irrigated conditions. Indian Journal of Agronomy 51: 283-287.

Gomez KA, Gomez AA (2010) Statistical Procedure for Agricultural Research, Second Ed. Wiley India (P) Ltd., New Delhi, Pp. 680.

Gopal R, Jat RK, Malik RK, Kumar V, Alam MM, Jat ML, Mazid MA, Saharawat YS, McDonald A, Gupta R (2010) Direct dryseeded rice production technology and weed management in rice based systems. Technical Bulletin. International Maize and Wheat Improvement Center, New Delhi, India, Pp. 28.

Jackson ML (1967) Soil Chemical Analysis. Prentice Hall of India, Pvt. Ltd., New Delhi, Pp. 111-203.

Jadhav KT, Lokhande DC, Asewa BV (2014) Effect of ferrous and zinc nutrient management practices on rice under aerobic condition. Advance Research Journal of Crop Improvement 5: 131-135.

Khush GS (2004) Harnessing science and technology for sustainable rice-based production systems. International Rice Commission Newsletter, FAO, Rome, 53: 17-18.

Kiran BO, Amaregouda A, Patil RP (2015) Effect of planting methods and nitrogen levels on tissue nitrogen content, yield and yield components in rice cultivars. Research Journal of Agricultural Science 6: 579-582.

Lindsay WL, Norvell WA (1978) Development of a DTPA soil test for zinc, iron, manganese and copper. Soil Science Society of American Journal 42: 421: 428

Piper CS (1966) Soil and Plant Analysis. Academic Press, New York, Pp. 367.

Rekha B, Jayadeva HM, Kombali G, Nagaraju GB, Mallikarjuna, Geethakumari A (2015) Growth and yield of aerobic rice grown under drip fertigation. The Ecoscan 9 : 435-437.

Subbaiah BY, Asija GL (1956) A rapid procedure for the estimation of available nitrogen in soils. Current Science 25: 259-260.

Yadav A, Singh DK, Sumit C, Kumar A, Nath A (2017) Growth and yield attributes of direct seeded aerobic rice (Oryza sativa L.) as influenced by seed rate and varieties. International Journal of Current Microbiology and Applied Science 6: 868-873.

Zhao DL, Atlin GN, Bastiaans L, Spiertz JHJ (2007) Cultivar weed competitiveness in aerobic rice: Heritability, correlated traits, and the potential for indirect selection in weed-free environment. Crop Science 46: 372-380. 